\title{
Synthesis and Characterization of Block Copolymers of Poly(ethylene oxide) and Poly(methyl methacrylate)
}

\author{
Toshimitsu Suzuki, Yoshimasa Murakami, and Yoshinobu TaKegami \\ Department of Hydrocarbon Chemistry, Faculty of Engineering, \\ Kyoto University, Kyoto 606, Japan.
}

(Received November 16, 1979)

\begin{abstract}
Anionic polymerization of methyl methacrylate (MMA) was initiated with the disodium salt of poly(ethylene oxide) (PEO) in tetrahydrofuran (THF) in both the presence and absence of a crown ether or cryptate. The resulting block copolymer of poly(methyl methacrylate) (PMMA) and PEO was characterized by ${ }^{1} \mathrm{H}$ - and ${ }^{13} \mathrm{C}-\mathrm{NMR}$ spectroscopies, gel-permeation chromatography and solvent extractions. The transesterification reaction between $\mathrm{PEO}$ and methoxy group in MMA was accompanied by polymerization, resulting in a PEO grafted triblock copolymer. A benzylamino derivative of PEO (PEO-N) was prepared as a new initiator system for the polymerization of MMA. The initiation with disodium or dilithium amide of PEO-N resulted in a linear triblock copolymer of PMMA-PEO-PMMA having a unimodal and narrower molecular weight distribution. The stereosequence distributions of the PMMA part in the copolymers were essentially atactic and the addition of the crown ether or the cryptate increased the fraction of the rr triad.
\end{abstract}

KEY WORDS Anionic Polymerization / Methyl Methacrylate / Poly(ethylene oxide) / Alkali Metal Alkoxide / Alkali Metal Amide / Crown Ether / Cryptate / Triblock Copolymer / Nuclear Magnetic Resonance /

The alkali metal salts of primary alcohols, except lithium $t$-butoxide, have been reported not to initiate the anionic polymerization of methyl methacrylate (MMA) ${ }^{1,2}$ However, sodium $n$-butoxide can initiate the polymerization of MMA in the presence of aprotic dipolar solvents such as hexamethylphosphoramide or dimethylsulfoxide. ${ }^{3}$ If an alkali metal salt of poly(ethylene oxide) (PEO), which is a long chained bifunctional primary alcohol, can initiate the polymerization of MMA, an A-B-A type triblock copolymer of poly(methyl methacrylate) (PMMA) and PEO will be obtained. Such a block copolymer, in which both the hydrophobic, glassy segment, and the hydrophilic, semicrystalline segment are contained, may represent useful characteristics, e.g., surface-active agents, highimpact plastics. Several attempts have been reported to obtain block copolymers of PEO and PMMA by radical polymerization. ${ }^{4,5}$ In anionic polymerization, such a block copolymer was obtained by initiating the polymerization of MMA with disodium salt of PEO in the presence of hexamethylphosphoramide. ${ }^{6}$ However, it is reported that a high-molecular-weight PEO did not initiate the polymerization of MMA and the yields of benzene-soluble polymer were low, accompanying gelation. Macrocyclic polyethers (crown ethers) have recently been known to bind effectively alkali metal cations and to increase the nucleophilicities of counter anions. ${ }^{7-10}$ More recently, macroheterobicycles (cryptates) have been found to be more effective in binding alkali metal cations than crown ethers. ${ }^{11-15}$ We have briefly reported the synthesis of a triblock copolymer of PMMA-PEOPMMA initiated with the disodium salt of PEO in the presence of a crown ether and a new initiator system. ${ }^{16,17}$

In this paper, we discuss the detailed nature of the polymerization of MMA prepared with disodium salt of PEO.

\section{EXPERIMENTAL}

\section{Materials}

Tetrahydrofuran (THF) and benzene were distilled twice over $\mathrm{LiAlH}_{4}$ under a nitrogen 
atmosphere and stored over molecular sieves $4 \mathrm{~A}$. Commercial MMA was purified by the usual method and distilled over calcium hydride at reduced pressure and stored over molecular sieves 4A. $t$-Butyl methacrylate (TBMA) was synthesized from methacrylic acid and isobutylene under acidic conditions. ${ }^{18}$ Sodium naphthalene and lithium naphthalene were prepared in THF by the usual methods. Dicyclohexyl-18-crown-6 (Nakarai Chemical), and kryptofix [222] 4,7,13,16,21,24hexaoxa-1, 10-diazabicyclo(8, 7, 8)hexacosane (Merck) were freeze-dried from a benzene solution and stored as benzene solution. Commercial PEO\#1000 $\quad\left(M_{n}=980, \quad M_{w} / M_{n}=1.06\right) \quad$ and PEO\#4000 $\left(M_{n}=3600, M_{w} / M_{n}=1.03\right)$ were freezedried from a benzene solution before use. The benzylamino derivatives of PEO (designated as PEO-N) were synthesized by the following scheme. $^{17}$
A reaction mixture was precipitated with a mixture of ether and petroleum ether $(1: 2$ volume) followed by washing with a small amount of benzene. From the benzene solution, PEO-N was recovered freezedrying. The uv absorption traces of gel-permeation chromatograms (GPC) of PEO-Ts and PEO-N were symmetrical and appeared strongly at a slightly smaller elution volume as compared with that of PEO. The molecular weight measured by VPO $\left(M_{n}=1140\right)$ was also in good agreement with that expected $\left(M_{n}=1192\right)$. The formation of PEO-N was ascertained by these findings together with elemental analyses. Anal. (PEO\#1000) Calcd for I, where $n \fallingdotseq 22, \mathrm{C}_{59} \mathrm{H}_{107} \mathrm{O}_{22} \mathrm{~N}_{2}: \mathrm{C}, 58.8 \% ; \mathrm{H}, 8.95 \% ; \mathrm{N}$, $2.34 \%$. Found: C, $59.2 \% ; \mathrm{H}, 8.88 \%$; N, $2.11 \%$. Anal. (PEO\#4000) Calcd for I, where $n \fallingdotseq 82$, $\mathrm{C}_{178} \mathrm{H}_{345} \mathrm{O}_{82} \mathrm{~N}_{2}: \mathrm{C}, 55.8 \% ; \mathrm{H}, 9.11 \% ; \mathrm{N}, 0.73 \%$. Found: $\mathrm{C}, 55.84 \%$; H, $9.16 \%$; N, $0.80 \%$. $\left.\mathrm{HO}+\mathrm{CH}_{2} \mathrm{CH}_{2} \mathrm{O}-\gamma_{n} \mathrm{H} \frac{\text { TsCl }}{\begin{array}{l}\text { Dry pyridine } \\ 4 \text { days, } 0^{\circ} \mathrm{C}\end{array}} \mathrm{TsO}-\mathrm{CH}_{2} \mathrm{CH}_{2} \mathrm{O}\right)_{n} \mathrm{Ts}$
$\left.\frac{\text { Excess } \mathrm{PhCH}_{2} \mathrm{NH}_{2}}{2 \text { days, } 30^{\circ} \mathrm{C}} \quad \mathrm{PhCH}_{2} \mathrm{NH}+\mathrm{CH}_{2} \mathrm{CH}_{2} \mathrm{O}\right)_{\overline{n-1}} \mathrm{CH}_{2} \mathrm{CH}_{2} \mathrm{NHCH}_{2} \mathrm{Ph}$

I

\section{Polymerization of $M M A$}

The polymerization of MMA was carried out in a three necked flask under an argon atmosphere. A certain amount of PEO or PEO-N was introduced into the flask and freeze-dried from the benzene solution for five hours under vacuum. Then, THF was distilled directly into the flask under vacuum. After being flushed with argon and cooled to $0^{\circ} \mathrm{C}$, a calculated amount of sodium naphthalene or lithium naphthalene was added. A calculated amount of benzene solution of a crown ether or cryptate was added to this solution at $30^{\circ} \mathrm{C}$. After an hour, a certain amount of MMA was introduced at $30^{\circ} \mathrm{C}$. After a given time, polymerization was stopped by adding $2 \mathrm{ml}$ of 2-propanol containing a small amount of hydrochloric acid. The reaction mixture was concentrated to one-third of its volume and poured into a large amount of petroleum ether. The polymer was filtered off, washed with petroleum ether and dried in vacuo at room temperature. In some cases, the polymers were fractionated with the acetone-methanol system: polymer, $0.3 \mathrm{~g}$; acetone, $5 \mathrm{ml}$; methanol, $50 \mathrm{ml}$.
(PEO-N)

\section{Polymerization of TBMA}

Polymerization of TBMA with the disodium salt of PEO was carried out in a similar manner as that of MMA in the presence of a cryptate.

\section{Transesterification Reaction Experiment}

Into a THF solution of the disodium salt of PEO (PEO $\# 4000,0.18 \mathrm{~g}$ ) in the presence of a crown ether $(0.1 \mathrm{mmol})$, a THF solution of anionically prepared PMMA $(0.70 \mathrm{~g})$ was introduced. The resulting solution was made to stand for 16 hours at $30^{\circ} \mathrm{C}$ and then poured into methanol. The precipitate was filtered off $(0.62 \mathrm{~g})$ and the filtrate was evaporated to dryness. The residue was washed repeatedly with water to remove free PEO, and dried $(0.11 \mathrm{~g})$. Both fractions were weighed and analyzed with the aid of infrared spectrometry and GPC.

\section{Characterization of the Copolymers}

Gel-permeation chromatograms (GPC) were recorded on a Waters ALC/GPC 244, equipped with four $\mu$-styragel columns $\left(10^{5}+10^{4}+10^{3}+500 \AA\right)$

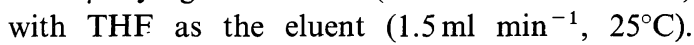
Standard polystyrenes were used for calibration. The 
weight-average molecular weight $\left(M_{w}\right)$ and numberaverage molecular weight $\left(M_{n}\right)$ of the polymers were estimated from their GPC traces. The true molecular weights of the polymers may somewhat deviate from those of standard polystyrenes due to the differences in their hydrodynamic volumes.

${ }^{13} \mathrm{C}$ NMR spectra were recorded in the Pulse Fourier Transformation mode at $25.0 \mathrm{MHz}$ on a JEOL-FX100 and ${ }^{1} \mathrm{H}$ NMR spectra were obtained on a Varian HR-220, using $\mathrm{CDCl}_{3}$ solutions of the polymers at an ambient temperature. Stereosequence distributions of the PMMA portion in the copolymers were determined up to pentad by carbonyl carbon resonances, according to the assignment by Chujo et al. ${ }^{19}$

\section{RESULTS AND DISCUSSION}

\section{Initiation with Disodium Salt of PEO}

The results are summarized in Table I. In this Table, the calculated molecular weights $\left(M_{n}\right)$ were simply calculated from the monomer to the initiator feed mole ratios $(\mathrm{M} / \mathrm{I})$. Since a $100 \%$ yield was obtained following ten minutes of polymerization in the presence of a crown ether as shown in No. 3, reaction times in the series of experiments are considered to have no significant meaning. Generally, the polymerizations are considered to proceed to completion within an hour.

In previous papers, the authors suggested that polymers prepared in this manner were not an admixture of PEO and PMMA. ${ }^{16,17}$ This was confirmed by solvent fractionation techniques and also by the fact that the peaks due to the unreacted PEO could not be observed in GPC traces of the copolymers. Although a $91 \%$ yield was adventituously obtained in No. 1, the yields of the copolymers prepared in the absence of the crown ether were generally lower than those in the presence of the crown ether (about $76 \%$ as shown in No. 7 ). When the crown ether was added to this polymerization system, high yields were usually obtained $(90-100 \%)$. As shown in the literatures, RONa cannot initiate the polymerization of MMA in the absence of the complexation reagents. ${ }^{1-3}$ The disodium salt of PEO, however, could initiate the polymerization of MMA, indicating that the nucleophilicity of alkoxy anions of PEO is somehow increased by the complexation of counter cations $\left(\mathrm{Na}^{+}\right)$with the PEO chain itself. It has been reported that PEO has a similar complexation capability toward alkali metal cations as crown ethers. ${ }^{20,21}$ However, judging from the yields of copolymers and the stereosequence distributions of PMMA part in the copolymers described below, the cation binding ability of PEO is considered to be inferior to that of a crown ether in our experiments. In addition, there was no difference in the ability of PEO\#1000 and $\# 4000$ to initiate the anionic polymerization of MMA.

\section{Transesterification Reaction}

In a previous paper, we suggested the possibility of a transesterification reaction between the disodium salt of PEO and the methoxy group of the PMMA portion in the copolymer or MMA monomer from ${ }^{1} \mathrm{H}$ NMR analyses. ${ }^{16}$

Since unreacted PEO could be contained in the copolymer recovering process, the peaks due to $\mathrm{PEO}$ should have appeared in GPC traces, occasional unreacted PEO in the reaction system. Figure 1 shows the GPC traces of the copolymers prepared in the presence of the crown ether at different $\mathrm{M} / \mathrm{I}$ ratios. Since no peaks due to $\mathrm{PEO}$ could be observed in Figure 1, no free PEO was contained in the recovered copolymers. PEO which did not initiate the polymerization of MMA is considered to have been exchanged with the ester groups of the MMA monomer or the PMMA part in the copolymers and incorporated into the copolymers as graft blocks. The transesterification reaction between PMMA and

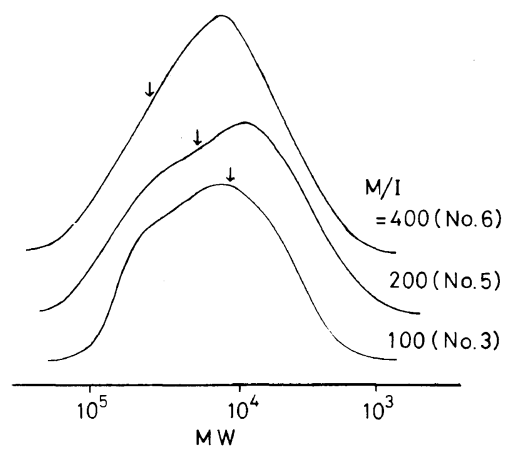

Figure 1. Gel-permeation chromatograms of PEOblock-PMMA (PEO- $b$-PMMA) prepared in the presence of a crown ether at different MMA monomer to PEO feed mole $\operatorname{ratios}(\mathrm{M} / \mathrm{I}): \mathrm{M} / \mathrm{I}=100$ (Run No. 3 in Table I), 200 (No. 5) and 400 (No. 6). $\downarrow$ indicates calculated $M_{n}$ from the values of $\mathrm{M} / \mathrm{I}$. 
dipotassium salt of PEO in toluene at a higher temperature $\left(110^{\circ} \mathrm{C}\right)$ has been reported. ${ }^{22}$ In our transesterification reaction experiment between anionically prepared PMMA and disodium salt of PEO in the presence of the crown ether, only about $5 \mathrm{wt} \%$ of PEO was incorporated into the PMMA. The absorption of the ether linkage $(\nu \mathrm{C}-\mathrm{O}-\mathrm{C}$ at 1110 $\mathrm{cm}^{-1}$ ) did not appear in the methanol-insoluble fraction from the transesterification reaction, but absorptions of the ether linkage and those of the ester group $\left(v_{\mathrm{C}=\mathrm{O}}\right.$ at $\left.1720 \mathrm{~cm}^{-1}\right)$ could be observed in the methanol-soluble and water-insoluble fractions. The shift of the molecular weight distributions (MWD) to the higher-molecular-weight region was not observed in the GPC traces of these two fractions. It was found from these results that the transesterification reaction between the PMMA part in the copolymers and the disodium salt of PEO in the presence of the crown ether did occur at $30^{\circ} \mathrm{C}$ but that only a small amount of methyl ester group was transesterified with PEO.

A terminal olefin proton resonance at $\delta 5.83 \mathrm{ppm}$ and a $\alpha$-methyl proton resonance at $\delta 1.95 \mathrm{ppm}$ were observed in ${ }^{1} \mathrm{H}$ NMR spectrum of unreacted PEO recovered and so the transesterification reaction is considered to occur between the MMA monomer and the disodium salt of PEO.* This reaction results in the formation of a new monomer which could possibly be copolymerized in this polymerization system. This new monomer indicated as $\mathrm{CH}_{2}=\mathrm{C}\left(\mathrm{CH}_{3}\right) \mathrm{COO}-\mathrm{PEO}$, must have brought about a large increase in molecular weight and a broadning of the MWD of the copolymer. Naturally, a transesterification reaction between the PMMA part in the copolymers and the disodium salt of PEO does occur to a small extent.

Figure 2 shows the MWDs of the copolymers prepared at different ratios of the crown ether to the initiator (Crown/I). As shown in Figure 2, with the addition of the crown ether, the MWDs of the copolymers shift to the higher molecular weight region. This indicates that the addition of the crown ether promotes not only the initiation reaction of the polymerization but also the transesterification reaction. In this polymerization, very little THF-or

* In certain cases, as a result of contamination to the reaction system and insufficient purification of THF or PEO, polymerization could not proceed at all, and the unreacted $\mathrm{PEO}$ was recovered.

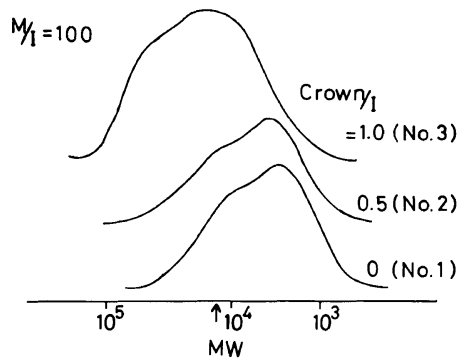

Figure 2. Gel-permeation chromatograms of PEO- $b$ PMMA prepared at different crown ether to PEO feed mole ratios (Crown/I): Crown/I $=0$ (Run No. 1 in Table I), 0.5 (No. 2) and 1.0 (No. 3). $\rightarrow$ indicates calculated $M_{n}$ from the value of $M / I$.

chloroform-insoluble polymers were obtained, indicating that the transesterification reaction to PMMA on both chain ends of PEO is negligible. However, the addition of a cryptate having stronger cation-binding properties than crown ethers ${ }^{11}$ resulted in instantaneous polymerization and gelation.

An acetone solution of the copolymer was reprecipitated into methanol, and the acetonemethanol-soluble $(22 \%)$ and-insoluble $(78 \%)$ fractions were recovered. If the transesterification reaction occurs very frequently, higher-molecularweight copolymers are incorporated into the acetone-methanol-soluble fraction, due to the solubility of large amounts of the PEO groups. Figure 3 shows GPC traces of the acetone-

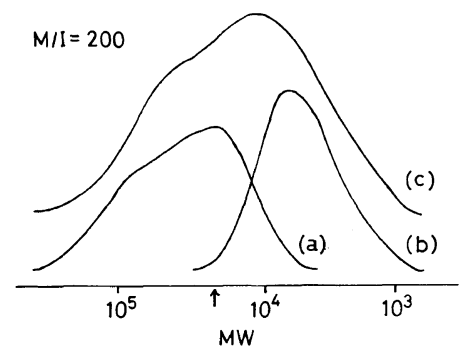

Figure 3. Gel-permeation chromatograms of PEO- $b$ PMMA (Run No. 5 in Table I) prepared in the presence of a crown ether: acetone-methanol (a) insoluble, (b) soluble fractions and (c) unfractionated copolymer. $\uparrow$ indicates calculated $M_{n}$ from the value of $\mathrm{M} / \mathrm{I}$. 
Table I. Polymerization of MMA with disodium salt of PEO

\begin{tabular}{|c|c|c|c|c|c|c|c|c|c|c|}
\hline \multirow{2}{*}{ No. } & \multirow{2}{*}{ PEO } & \multirow{2}{*}{$\mathrm{M} / \mathrm{I}^{\mathrm{b}}$} & \multirow{2}{*}{ Crown $/ \mathbf{I}^{\mathbf{c}}$} & \multirow{2}{*}{$\frac{\text { Time }}{h}$} & \multirow{2}{*}{$\frac{\text { Yield }^{d}}{w t^{o} \%}$} & \multirow{2}{*}{$\frac{\text { Calculated }^{\mathrm{e}}}{M_{n} \times 10^{-3}}$} & \multicolumn{2}{|c|}{ Observed $^{\mathrm{f}}$} & \multirow{2}{*}{$M_{w} / M_{n}$} & \multirow{2}{*}{$\mathrm{MMA} / \mathrm{PEO}^{\circ}$} \\
\hline & & & & & & & $M_{w} \times 10^{-3}$ & $M_{n} \times 10^{-3}$ & & \\
\hline 1 & \multirow[t]{6}{*}{$\# 1000$} & 100 & 0 & 17 & 91 & 11.0 & 6.5 & 2.9 & 2.25 & - \\
\hline 2 & & 100 & 0.5 & 17 & 93 & 11.0 & 8.0 & 3.6 & 2.19 & - \\
\hline 3 & & 100 & 1.0 & 0.2 & 100 & 11.0 & 20.6 & 9.1 & 2.27 & 67 \\
\hline 4 & & 40 & 1.0 & 1 & 98 & 5.0 & 4.1 & 2.3 & 1.81 & 31 \\
\hline 5 & & 200 & 1.0 & 1 & 98 & 21.0 & 21.7 & 6.2 & 3.51 & 128 \\
\hline 6 & & 400 & 1.0 & 1 & 90 & 41.0 & 26.2 & 10.4 & 2.52 & - \\
\hline 7 & \multirow[t]{4}{*}{$\# 4000$} & 200 & 0 & 20 & 76 & 23.6 & 19.4 & 5.0 & 3.88 & - \\
\hline 8 & & 100 & 1.0 & 1.5 & 96 & 13.6 & 25.6 & 4.5 & 5.64 & 86 \\
\hline 9 & & 200 & 1.0 & 20 & 97 & 23.6 & 21.4 & 7.2 & 2.97 & - \\
\hline 10 & & 400 & 1.0 & 15 & 93 & 43.6 & 37.7 & 13.8 & 2.74 & - \\
\hline
\end{tabular}

a In THF at $30^{\circ} \mathrm{C}$.

b MMA to PEO feed mole ratio.

c Crown ether to PEO feed mole ratio.

d Against (PEO+MMA) charged.

e Calculated from the value of $\mathrm{M} / \mathrm{I}$

${ }^{f}$ Estimated from GPC traces calibrated with standard polystyrenes.

${ }^{g}$ MMA monomer units/PEO; obtained by ${ }^{1} \mathrm{H}$ NMR spectrometries. 
methanol-soluble and insoluble fractions of copolymer No. 5. A simple fractionation due to differences in molecular weight is observed; that is, the lower molecular weight fraction, was recovered from the soluble fraction. The ratio of the MMA monomer units to one PEO chain (MMA/PEO) obtained by ${ }^{1} \mathrm{H}$ NMR spectra are listed in the last column of Table I. The values of MMA/PEO of both fractions are not significantly different from that of the unfractionated copolymer (soluble fraction, 105; insoluble fraction, 133; unfractionated copolymer, 128). The broad GPC trace of the insoluble fraction appeared in a molecular weight ranging from ten thousand to one hundred thousand. If the insoluble fraction contains only one initiator fragment, the ratio of MMA/PEO will be very large.

As shown in Figures 1 and 2, fairly broad MWDs are observed, compared to those of polymers prepared by general anionic polymerization. As described above, the broadning of MWDs and the existence of appreciably high-molecular-weight copolymers are due mainly to the transesterification reaction between the disodium salt of PEO and a methoxy group of the MMA monomer. Another important factor for this may be the slower rate of the initiation reaction compared to that of the propagation reaction.

\section{Polymerization of TBMA with Disodium Salt of PEO}

The transesterification reaction of $t$-butyl ester does not occur under basic conditions. The polymerization of TBMA with the disodium salt of PEO\#1000 in the presence of a cryptate (kryptofix[222]) at $30^{\circ} \mathrm{C}$ gave a copolymer with a $100 \%$ yield; $94 \%$ of this was soluble in petroleum ether and $6 \%$, insoluble. Infrared spectra indicated that both fractions are copolymers of PEO and poly ( $t$-butyl methacrylate) (PTBMA). The TBMA/PEO ratio of petroleum ether-soluble fraction obtained by ${ }^{1} \mathrm{H}$ NMR analysis showed good agreement with the $\mathrm{M} / \mathrm{I}$ ratio (TBMA $/ \mathrm{PEO}=57, \mathrm{M} / \mathrm{I}=54$ ).

Figure 4 shows the GPC traces of the petroleum ether-soluble and insoluble fractions of the copolymer (soluble: $M_{w}=18500, \quad M_{n}=9800$, $M_{w} / M_{n}=1.89$; insoluble: $M_{w}=44300, M_{n}=38200$, $\left.M_{w} / M_{n}=1.16\right)$. As shown in Figure 4, the MWDs of each fraction are relatively narrow and almost unimodal. Thus in the polymerization of TBMA, the transesterification reaction did not occur even in the presence of the cryptate resulting in instantaneous

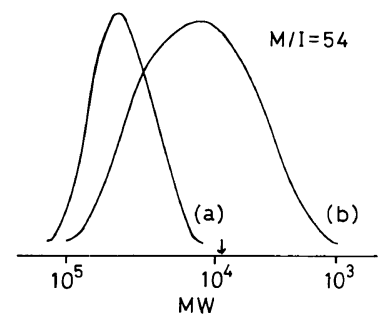

Figure 4. Gel-permeation chromatograms of PEO- $b$ PTBMA prepared in the presence of a cryptate: petroleum ether (a) insoluble and (b) soluble fractions. $\downarrow$ indicates calculated $M_{n}$ from the value of $\mathrm{M} / \mathrm{I}$.

gelation in the polymerization of MMA. This result strongly supports the consideration that in the polymerization of MMA, the existence of fairly highmolecular-weight copolymers and the broadening of the MWDs of the copolymers may be attributed to the transesterification reaction. In the polymerization of TBMA, the main product is considered to be a nongrafted linear A-B-A-type triblock copolymer of PTBMA-PEO-PTBMA.

\section{Initiation with Disodium and Dilithium Amides of PEO-N}

As previously reported, a new initiator of benzylamino derivative of PEO (PEO-N) was developed to reduce the extent of the transesterification reaction. ${ }^{17}$ Sodium or lithium amides of secondary amines are known to be good initiators for MMA. ${ }^{23}$ The results of the polymerization of MMA with disodium and dilithium amides of PEO-N are summarized in Table II. Although all attempts to initiate the polymerization of MMA using dilithium salt of PEO have been unsuccessful, dilithium amide of PEO-N initiated the polymerization of MMA, giving a block copolymer. As shown in Table II, the initiation with dilithium amide of PEO-N resulted in very low yields of copolymers compared with that obtained by the use of disodium amide, even in the presence of the cryptate. The effect of additives could not be distinctly detected in the case of dilithium amides. Since a considerable amount of PEO-N did not initiate the polymerization in the case of dilithium amides, the molecular weights of the copolymers were rather high. However, the values of $M_{w} / M_{n}$ for these copolymers (No. 17, 18, and 19) were relatively small. Therefore, the propagation reaction is considered to have proceeded uniformly. 
Table II. Polymerization of MMA with disodium and dilithium amides of PEO-N ${ }^{a}$

\begin{tabular}{|c|c|c|c|c|c|c|c|c|c|}
\hline \multirow{2}{*}{ No. } & \multirow{2}{*}{ PEO } & \multirow{2}{*}{$\begin{array}{c}\text { Counter } \\
\text { ion }\end{array}$} & \multirow{2}{*}{ Additive $^{b}$} & \multirow{2}{*}{$\frac{\text { Time }}{\mathrm{h}}$} & \multirow{2}{*}{$\begin{array}{l}\text { Yield }^{\mathrm{c}} \\
\mathrm{wt} \%\end{array}$} & \multirow{2}{*}{$\frac{\text { Calculated }^{\mathrm{d}}}{M_{n} \times 10^{-3}}$} & \multicolumn{2}{|c|}{ Observed $^{\mathrm{e}}$} & \multirow{2}{*}{$M_{w} / M_{n}$} \\
\hline & & & & & & & $M_{w} \times 10^{-3}$ & $M_{n} \times 10^{-3}$ & \\
\hline 11 & \multirow[t]{3}{*}{$\# 1000$} & $\mathrm{Na}^{+}$ & - & 15 & 92 & 19.2 & 20.5 & 12.0 & 1.71 \\
\hline 12 & & $\mathrm{Na}^{+}$ & Crown & 18 & 100 & 11.2 & 12.5 & 8.5 & 1.47 \\
\hline 13 & & $\mathrm{Na}^{+}$ & Cryptate & 14 & 100 & 11.2 & 66.0 & 24.8 & 2.66 \\
\hline 14 & \multirow[t]{3}{*}{$\# 4000$} & $\mathrm{Na}^{+}$ & 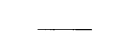 & 18 & 56 & 13.8 & 9.5 & 7.3 & 1.30 \\
\hline 15 & & $\mathrm{Na}^{+}$ & Crown & 18 & 83 & 13.8 & 12.6 & 9.9 & 1.28 \\
\hline 16 & & $\mathrm{Na}^{+}$ & Cryptate & 1 & 100 & 13.8 & 45.5 & 21.4 & 2.12 \\
\hline 17 & \multirow[t]{3}{*}{$\# 1000$} & $\mathrm{Li}^{+}$ & - & 17 & 30 & 11.2 & 25.5 & 13.3 & 1.92 \\
\hline 18 & & $\mathrm{Li}^{+}$ & Crown & 14 & 17 & 11.2 & 40.7 & 25.8 & 1.58 \\
\hline 19 & & $\mathrm{Li}^{+}$ & Cryptate & 19 & 37 & 11.2 & 29.1 & 16.0 & 1.82 \\
\hline
\end{tabular}

${ }^{\text {a }}$ In THF at $30^{\circ} \mathrm{C}$; MMA to PEO-N feed mole ratios $(\mathrm{M} / \mathrm{I})$ are 100 , except in No. $11(\mathrm{M} / \mathrm{I}=180)$.

${ }^{b}$ Crown, dicyclohexyl-18-crown-6; cryptate, Kryptofix [222].

c Against (PEO-N + MMA) charged.

d Calculated from the value of $\mathrm{M} / \mathrm{I}$.

e Estimated from the GPC traces calibrated with standard polystyrenes. 
In the case of the polymerization with disodium amides, the molecular weights of the copolymers, except those prepared in the presence of the cryptate, are fairly close to the calculated ones compared with the initiation by disodium salt of PEO, indicating the absence of a transesterification reaction. As shown in the last column of Table II, the values of $M_{w} / M_{n}$ are generally small. These are also shown in Figure 5, depicting the effect of additives on the MWDs of the copolymers. In contrast to the copolymers prepared with the disodium salt of PEO, the copolymers prepared with disodium amides of

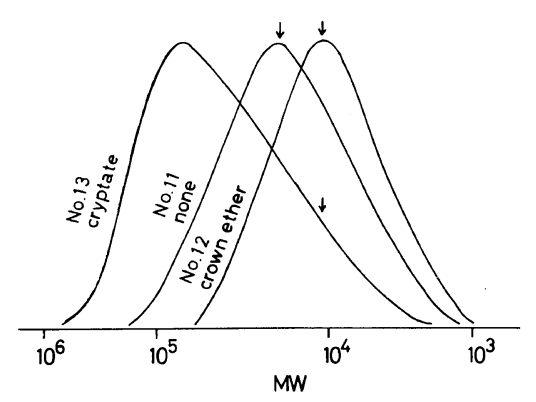

Figure 5. Gel-permeation chromatograms of PEO- $b$ PMMA prepared with disodium amide of PEO\#1000-N in the absence of the additive (Run No. 11 in Table II) and the presence of a crown ether (No. 12) or a cryptate (No. 13). $\downarrow$ indicates calculated $M_{n}$ from the values of $\mathrm{M} / \mathrm{I}$.
PEO-N have narrow and unimodal MWDs. As can be seen in Figure 5, extremely high-molecular-weight copolymers were obtained in the presence of the cryptate. The activation of anions by the cryptate may principally affect the growing polymer chain end anions rather than the initiator anions. The rate of the propagation reaction, therefore, was increased considerably, resulting in very high-molecularweight copolymers. From these results, it is shown that nongrafted linear triblock copolymers of PMMA-PEO-PMMA were synthesized with the disodium amides of PEO-N in the presence of the crown ether in THF. However, it is very difficult to obtain diamine derivatives of PEO having higher molecular weights, by the method described above.

\section{Stereosequence Distributions of PMMA Part in the Copolymers}

The observed and calculated triad stereosequence distributions of the PMMA part in the copolymers are summarized in Table III. As previously reported, in the polymerization of MMA by RONa in toluene in the presence of donor solvents, the nature of the donor solvent and the ratio of the donor to the initiator affect the stereosequence distributions. ${ }^{3}$

The Stereosequence distributions of the PMMA part in the copolymers prepared with the disodium salt of PEO in THF were essentially atactic. In general, an anionic polymerization of MMA in a

Table III. Stereosequence distributions of PMMA part in the copolymers

\begin{tabular}{|c|c|c|c|c|c|c|c|c|c|}
\hline \multirow{2}{*}{ No. } & \multirow{2}{*}{ PEO } & \multirow{2}{*}{$\begin{array}{c}\text { Counter } \\
\text { ion }\end{array}$} & \multirow{2}{*}{ Additive $^{a}$} & \multicolumn{3}{|c|}{ Observed $^{\mathrm{b}}$} & \multicolumn{3}{|c|}{ Calculated $^{\mathrm{c}}$} \\
\hline & & & & $\mathrm{mm}$ & $\mathrm{mr}+\mathrm{rm}$ & $\mathrm{rr}$ & $\mathrm{mm}$ & $\mathrm{mr}+\mathrm{rm}$ & $\mathrm{rr}$ \\
\hline 1 & $\# 1000$ & $\mathrm{Na}^{+}$ & - & 0.11 & 0.52 & 0.37 & 0.14 & 0.47 & 0.39 \\
\hline 5 & & $\mathrm{Na}^{+}$ & Crown & 0.10 & 0.44 & 0.46 & 0.10 & 0.44 & 0.46 \\
\hline 7 & $\# 4000$ & $\mathrm{Na}^{+}$ & - & 0.15 & 0.50 & 0.35 & 0.16 & 0.48 & 0.36 \\
\hline 10 & & $\mathrm{Na}^{+}$ & Crown & 0.10 & 0.43 & 0.47 & 0.10 & 0.43 & 0.47 \\
\hline 11 & $\# 1000-\mathrm{N}$ & $\mathrm{Na}^{+}$ & - & 0.14 & 0.53 & 0.33 & 0.16 & 0.48 & 0.36 \\
\hline 12 & & $\mathrm{Na}^{+}$ & Crown & 0.10 & 0.39 & 0.51 & 0.09 & 0.41 & 0.50 \\
\hline 13 & & $\mathrm{Na}^{+}$ & Cryptate & 0.07 & 0.39 & 0.54 & 0.07 & 0.39 & 0.54 \\
\hline 17 & & $\mathrm{Li}^{+}$ & 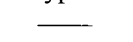 & 0.09 & 0.37 & 0.54 & 0.07 & 0.40 & 0.53 \\
\hline 19 & & $\mathrm{Li}^{+}$ & Cryptate & 0.06 & 0.36 & 0.58 & 0.06 & 0.36 & 0.58 \\
\hline
\end{tabular}

a Crown, dicyclohexyl-18-crown-6; cryptate, Kryptofix [222].

b Observed values from ${ }^{13} \mathrm{C}$ NMR spectra.

c Calculated values based on the Bernoullian statistics. 
polar solvent is considered to proceed in a free ionic manner to give a syndiotactic polymer $(\mathrm{rr} \simeq 0.60) .{ }^{24}$ It is also well-known that fluorenyl sodium exists in THF as an equilibrium mixture of contact and solvent separated ion pairs at room temperature. ${ }^{25}$ Thus in the case of sodium as counter ions the fairly small amount of $\mathrm{rr}$ triads in the absence of additives (No. 1, 7, and 11) can be accounted for by the association of sodium counter ions with the carbonyl groups in living chain ends. As reported in a previous paper, in the absence of the crown ether, the triad stereosequence distributions deviated from Bernoullian statistics. ${ }^{16}$ However, the polymers shown in No. 7 are well-defined by Bernoullian statistics. Since it is difficult to explain systematically this result, the pentad stereosequence distributions were obtained using ${ }^{13} \mathrm{C}$ NMR spectra to obtain more detailed information on the stereosequence distributions of the polymers. The triad stereosequence distributions of the copolymer No. 11 is not in good agreement with that calculated from Bernoullian statistics. The first-order Markov chain parameters of this polymer were obtained using triad-dyad relations $\left(P_{\mathrm{m} / \mathrm{m}}=0.346, \quad P_{\mathrm{m} / \mathrm{r}}=0.413\right.$, $P_{\mathrm{r} / \mathrm{m}}=0.654$, and $\left.P_{\mathrm{r} / \mathrm{r}}=0.586\right)$. The pentad stereosequence distributions calculated from these parameters are in fairly good agreement with those observed by ${ }^{13} \mathrm{C}$ NMR spectroscopy: $\mathrm{mmmm}=$ 0.021 (observed) [0.016(first-order Markov)], $\mathrm{mmmr}=0.059 \quad[0.060], \quad \mathrm{rmmr}=0.053 \quad[0.057]$, $\mathrm{mmrm}+\mathrm{rmrm}=0.221[0.209], \mathrm{mmrr}+\mathrm{rmrr}=0.285$ [0.294], $\mathrm{mrrm}=0.079[0.061], \mathrm{rrrm}=0.158[0.173]$, and $\operatorname{rrrr}=0.122[0.123]$.

Since the value of $P_{\mathrm{m} / \mathrm{r}}+P_{\mathrm{r} / \mathrm{m}}(=1.07)$ is very close to 1 , the calculated stereosequence distributions from a first-order Markov chain model and Bernoullian statistics are in the same order of magnitude. Therefore, not only a solvent separated and a contact ion pairs but also an agent (PEO) separated ion pair are considered to coexist in the polymerization system in the absence of the additives. The uncertainty in the stereosequence distributions in the absence of the additives will be interpreted by the combinations of the structures which are easily affected by the conditions of the polymerization.

In the presence of the crown ether or the cryptate, the fractions of the rr triads increased significantly and the triad stereosequence distributions are in good agreement with those calculated from
Bernoullian statistics; that is, a metal cation was captured by the crown ether or the cryptate in a propagating chain end to form predominantly an agent (crown ether or cryptate) separated ion pair.

In the case of lithium as a counter ion, the fraction of the rr triad increased even in the absence of the additives. In this case, the polymerization of MMA is considered to proceed mainly in a solvent separated ion pair.

\section{CONCLUSION}

In the polymerization of MMA with the disodium salt of PEO, a block copolymer having a broad and non-unimodal MWD was obtained. The broadening of the MWD of the copolymer was partly attributed to the transesterification reaction and, consequently, a PEO grafted A-B-A-type triblock copolymer was obtained.

In the polymerization of TBMA with the disodium salt of PEO in the presence of the cryptate, a nongrafted linear triblock copolymer of PTBMA-PEOPTBMA was obtained.

The initiation of the polymerization of MMA with disodium or dilithium amides of PEO-N provided a nongrafted linear triblock copolymer of PMMA-PEO-PMMA whose MWD was relatively narrow and unimodal.

Stereosequence distributions of the PMMA part in the copolymers were essentially atactic. The addition of the crown ether or the cryptate to the polymerization system increased the fractions of the rr triads.

\section{REFERENCES}

1. N. S. Wooding and W. C. E. Higginson, J. Chem. Soc., 774 (1952).

2. J. Trekoval, J. Polym. Sci., A-1, 9, 2575 (1971).

3. M. Tomoi, K. Sekiya, and H. Kakiuchi, Polym. J., 6, 438 (1974).

4. Y. Minoura, T. Kasuya, S. Kawamura, and A. Nakano, J. Polym. Sci., A-1, 5, 43 (1967).

5. J. Galin, M. Galin, and P. Calme, Makromol Chem., 134, 273 (1970).

6. M. Tomoi, Y. Shibayama, and H. Kakiuchi, Polym. J., 8, 190 (1976).

7. J. Smid, Angew. Chem. Int. Ed., 11, 112 (1972).

8. S. Kopolov, T. E. Hogen-Esch, and J. Smid, Macromolecules, 6, 133 (1973).

9. H. D. Durst, Tetrahedron Lett., 2421 (1974).

10. C. L. Liotta, E. E. Grisdale, and H. P. Hophins, Jr., Tetrahedron Lett., 4205 (1975). 
11. J. M. Lehn, B. Dietrich, J. P. Sauvage, and J. Blanzat, Tetrahedron, 29, 1629 (1973).

12. J. M. Lehn, B. Dietrich, and J. P. Sauvage, Tetrahedron, 29, 1647 (1973).

13. B. Dietrich and J. M. Lehn, Tetrahedron Lett., 1225 (1973).

14. S. Boileau, B. Kaempf, J. M. Lehn, and F. Schué, $J$. Polym. Sci., Polym. Lett. Ed., 12, 203 (1974).

15. J. Lacoste, F. Schué, S. Bywater, and B. Kaempf, $J$. Polym. Sci., Polym. Lett. Ed., 14, 201 (1976).

16. T. Suzuki, Y. Murakami, Y. Tsuji, and Y. Takegami, J. Polym. Sci., Polym. Lett. Ed., 14, 675 (1976).

17. T. Suzuki, Y. Murakami, and Y. Takegami, J. Polym. Sci., Polym. Lett. Ed., 17, 241 (1979).

18. A. L. McCloskey, G. S. Fonken, R. W. Kluiber, and W. S. Johnson, "Organic Syntheses," Collective Vol.
4, N. Rabjohn, Ed., Wiley, New York, N.Y., 1963, p 261.

19. Y. Inoue, A. Nishioka, and R. Chujo, Polym. J., 4, 535 (1971).

20. I. M. Panayotov, C. B. Tsvetanov, and D. K. Dimov, Makromol. Chem., 177, 279 (1976).

21. S. Yanagida, K. Takahashi, and M. Okahara, Bull. Chem. Soc. Jpn., 50, 1386 (1977).

22. M. H. Twaik, M. Tahan, and A. Zilkha, J. Polym. Sci., $A-1,7,2469$ (1969).

23. T. Tsuruta, "Progress in Polymer Science, Japan," 3, Kodansha, Tokyo, 1972, p 1.

24. H. Yuki, K. Hatada, K. Ohta, and Y. Okamoto, J. Macromol. Sci.Chem., A9, 983 (1975).

25. T. E. Hogen-Esch and J. Smid, J. Am. Chem. Soc., 88, 307 (1966). 